\title{
Fear Extinction in Rodents
}

\author{
Chun-hui Chang, ${ }^{1}$ Ewelina Knapska, ${ }^{1}$ Caitlin A. Orsini, ${ }^{1}$ Christine A. \\ Rabinak, ${ }^{1}$ Joshua M. Zimmerman, ${ }^{1}$ and Stephen Maren ${ }^{1}$ \\ ${ }^{1}$ University of Michigan, Ann Arbor, Michigan
}

\begin{abstract}
Pavlovian conditioning paradigms have become important model systems for understanding the neuroscience of behavior. In particular, studies of the extinction of Pavlovian fear responses are yielding important information about the neural substrates of anxiety disorders, such as phobias and post-traumatic stress disorder (PTSD) in humans. These studies are germane to understanding the neural mechanisms underlying behavioral interventions that suppress fear, including exposure therapy in anxiety disorders. This unit describes detailed behavioral protocols for examining the nature and properties of fear extinction in laboratory rodents. Curr. Protoc. Neurosci. 47:8.23.1-8.23.17. (C) 2009 by John Wiley \& Sons, Inc.
\end{abstract}

Keywords: extinction $\bullet$ fear $\bullet$ rat $\bullet$ anxiety $\bullet$ phobia

\section{INTRODUCTION}

Extinction of classical fear conditioning refers to a reduction in conditional responding after the repeated presentation of a conditioned stimulus (CS, usually a tone) in the absence of the unconditioned stimulus (US, usually a footshock) with which it was previously paired (see UNIT $8.5 \mathrm{C}$ for fear conditioning procedures). Interestingly, extinguished fear can be recovered in a number of situations. It can re-emerge with the passage of time after extinction (spontaneous recovery), because of a change in experimental context (renewal effect), or from an unsignaled presentation of the US (reinstatement effect). These observations suggest that extinction is a new learning process, and the fear reduction results from inhibition rather than erasure of the original fear memory. Moreover, unlike fear conditioning, extinction is highly context-specific (for reference see Bouton et al., 2006; Ji and Maren 2007).

Because knowledge of the conditions that facilitate extinction learning may help to optimize extinction-based exposure therapies for the treatment of anxiety disorders, such as panic disorder and post-traumatic stress disorder (PTSD), the behavioral and neural mechanisms of fear extinction have attracted increasing attention over the last 20 years (Hermans et al., 2006). It has been shown that fear extinction engages several brain structures, including the amygdala, hippocampus, and prefrontal cortex (see Ji and Maren, 2007; Quirk and Mueller, 2008). Typical methods for conducting an extinction experiment in rats are presented below. Procedures for fear extinction are first described (see Basic Protocol), followed by alternative procedures including renewal (see Alternate Protocol 1), spontaneous recovery (see Alternate Protocol 2), and reinstatement (see Alternate Protocol 3).

NOTE: All protocols using live animals must first be reviewed and approved by an Institutional Animal Care and Use Committee (IACUC) and must follow officially approved procedures for the care and use of laboratory animals.

\section{STRATEGIC PLANNING}

The observation chambers $(30 \times 24 \times 21-\mathrm{cm}$; MED Associates $)$ are constructed of aluminum (side walls) and Plexiglas (rear wall, ceiling, and hinged front door) and are

Current Protocols in Neuroscience 8.23.1-8.23.17, April 2009

Published online April 2009 in Wiley Interscience (www.interscience.wiley.com).

DOI: 10.1002/0471142301.ns0823s47

Copyright $\subset 2009$ John Wiley \& Sons, Inc.
UNIT 8.23

Behavioral Neuroscience

\subsection{1}

Supplement 47 
situated in sound-attenuating cabinets located in a brightly lit and isolated room. The floor of each chamber consists of 19 stainless steel rods (4-mm in diameter) spaced $1.5-\mathrm{cm}$ apart (center-to-center). Rods are wired to a shock source and solid-state grid scrambler (MED Associates) for the delivery of footshock US (0.1 to $1.0 \mathrm{~mA}$ ). A speaker mounted outside a grating in one wall of the chamber is used for the delivery of acoustic CS (pure tones or white noise).

Each conditioning chamber rests on a load-cell platform that is used to record chamber displacement in response to each rat's motor activity. This load-cell platform is acquired online via Threshold-Activity software (MED Associates). The output of each chamber's load cell is set to a gain that is optimized for detecting freezing behavior (somatomotor immobility, except that necessitated by breathing). Load-cell amplifier output ( -10 to $+10 \mathrm{~V}$ ) from each chamber is digitized, and absolute values of the load-cell voltages are computed and multiplied by 10 to yield a scale that ranges from 0 to 100 . For each chamber, load-cell voltages are digitized at $5 \mathrm{~Hz}$, yielding one observation every $200 \mathrm{msec}$.

The observation chamber has a video camera mounted on the ceiling of the soundattenuating cabinet facing down into the chamber. Each camera is routed to Monochrome Simplex Multiplexer, which is connected to a VCR, and allows multiple video cameras to record onto one VCR. It is ideal to have the multiplexer and VCR located outside of the conditioning room so that experimenters can view the session live without interfering.

BASIC PROTOCOL

Fear Extinction in Rodents

8.23.2

\section{FEAR EXTINCTION IN RATS}

This protocol uses freezing behavior in rats to index the acquisition and extinction of learned fear. It is important to point out that the conditioning and extinction of fear exhibits similar properties in several species (e.g., rats, mice, rabbits, cats) using many different response measures (e.g., acoustic startle, bar press suppression, heart rate; Teich et al., 1989; Davis, 2001; Woods and Bouton, 2006). Any of these behavioral responses can be measured as an index of fear; however, freezing behavior is easily quantifiable and is thus used as a measure of fear for the purpose of this unit. Typically, extinction experiments require 8 to 16 subjects for appropriate statistical power. The protocol described here makes use of automated methods for assessing freezing behavior, although visual observation is a reliable and a less expensive alternative (but requires that observers be blind to the experimental conditions), both of which have been used in the authors' laboratory. The system described in this protocol was manufactured by MED Associates and makes use of force transducers underneath the conditioning chambers to

A

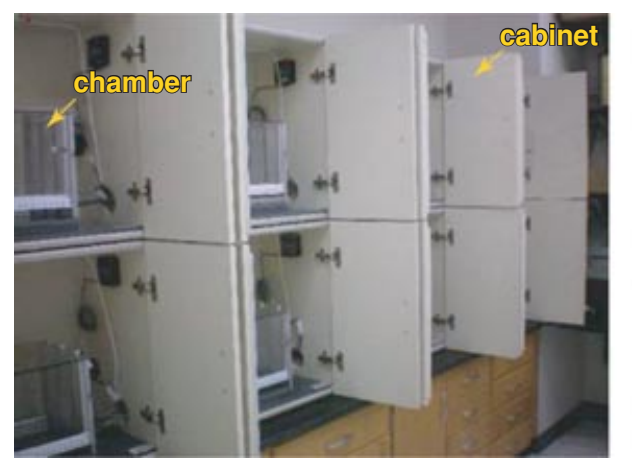

B

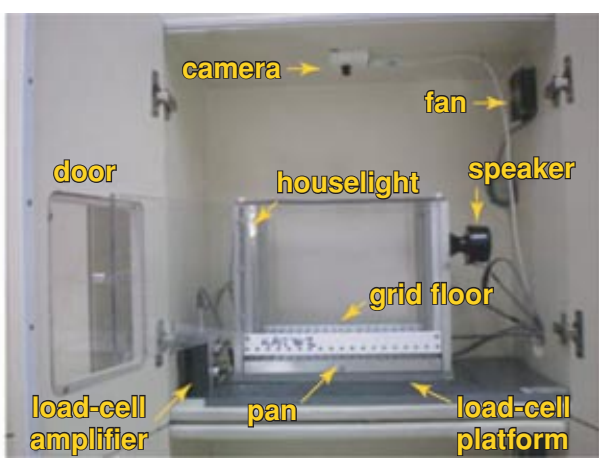

Figure 8.23.1 Photographs of typical equipment used for fear conditioning, extinction, and testing. (A) A set of eight experimental chambers (MED Associates) are situated in sound-attenuating cabinets and located in an isolated room. (B) Components of a typical conditioning chamber and cabinet. 
Table 8.23.1 Fear Extinction Experimental Design (Basic Protocol) ${ }^{a}$

\begin{tabular}{lccc}
\hline Group & Conditioning & Extinction & Test \\
\hline EXT & AT + & BT - & BT - \\
NO-EXT & AT + & B - & BT - \\
\hline${ }^{a}$ A and B refer to contexts; T $=$ auditory CS (tone); + and - indicate footshock or the \\
lack of footshock, respectively; EXT: extinction group, NO-EXT: no-extinction control.
\end{tabular}

detect motion in the experimental subjects (see Fig. 8.23.1). The behavioral sessions are commonly denoted by a three-letter code, with each letter corresponding to the context used for the conditioning, extinction, and testing sessions. A basic protocol for an ABB design (i.e., in which the context is the same during extinction and testing but different from the context in which fear is first acquired) is outlined here (Table 8.23.1). In this particular protocol, $24 \mathrm{hr}$ is interposed between each behavioral session and the entire protocol requires 3 days to complete.

\section{Materials}

$1 \%$ ammonium and $1 \%$ acetic acid solution for cleaning chambers (odors from these solutions are also used to establish unique olfactory contexts)

Rats (e.g., adult, male Long-Evans)

Med-Test software (MED Associates)

Shock calibration tool (MED Associates or equivalent) or an oscilloscope with alligator clips

A computer that will run MED-PC software and interface with the chambers for stimulus delivery and data acquisition

Decibel meter

Load-cell platforms (see Strategic Planning)

Observation/conditioning chambers $(30 \times 24 \times 21-\mathrm{cm}$; MED Associates; see Strategic Planning)

Video camera mounted on the ceiling of the sound-attenuating cabinet facing down into the observation chamber (see Strategic Planning)

White and black plastic boxes

\section{Create unique experimental contexts}

Two distinct contexts are used in this protocol (Fig. 8.23.2). For the first context (context A), a 15-W houselight mounted opposite the speaker is turned on, and room lights remain on. The chambers are cleaned with a $1 \%$ acetic acid solution. To provide a distinct odor, stainless steel pans containing a thin layer of this solution are placed underneath the grid floors before the rats are placed inside. Ventilation fans in each chest surrounding the chamber supplies background noise $(65 \mathrm{~dB})$. Rats are transported from their home cages to this context in white plastic boxes.

For the second context (context B), all room and chamber houselights are turned off. Red fluorescent lights are on, providing illumination. Additionally, the doors on the soundattenuating cabinets are closed, the ventilation fans are turned off, and the chambers are cleaned with $1 \%$ ammonium hydroxide solution. Also, stainless steel pans containing a thin layer of the same solution are placed underneath the grid floors before the rats are placed inside to provide a distinct odor. Rats are transported to this context in black plastic boxes.

\section{Day 1: Conditioning to an auditory CS in a novel context}

On day 1 the animals are conditioned to the auditory CS in context A.

1. Turn on all equipment.

Behavioral Neuroscience

8.23.3

Supplement 47 


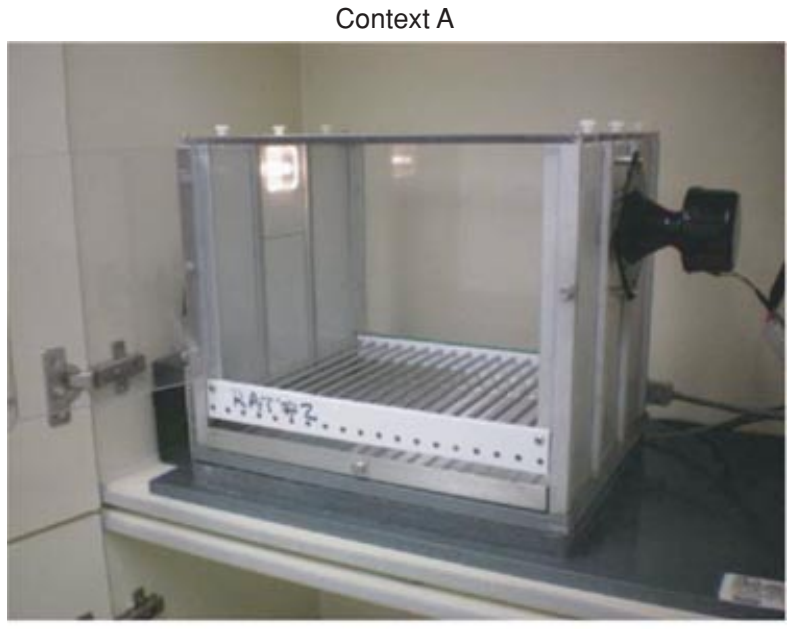

Context B

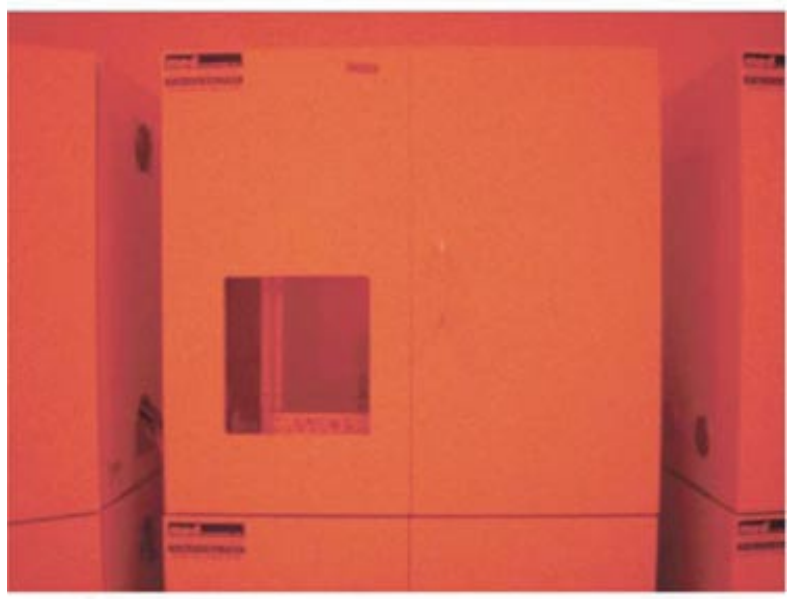

Context C

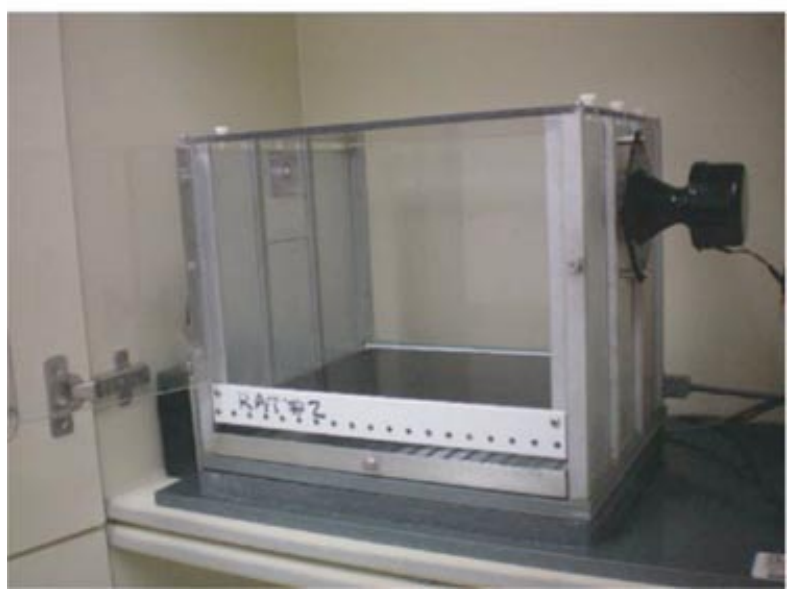

Figure 8.23.2 Representative photographs of each experimental context. The contexts differ not only visually, but also in terms of the olfactory, auditory, tactile, and transport cues (see text for examples of contextual properties that differ for each of the contexts). 
2. Calibrate the shock intensity. Calibrating the footshock intensity is essential as increased footshock intensity leads to increased levels of fear and, consequently, higher levels of freezing. Calibrating the footshock requires the Med-Test software and either a shock calibration tool (MED Associates or equivalent) or an oscilloscope with alligator clips. The Med-Test software will deliver a shock of selected intensity (current) to the grid of a given chamber. With the alligator clips connected to two different bars of the same grid floor, measure the actual shock current using the oscilloscope or the shock test tool. If the selected output and actual output differ, adjust the shock generator for that chamber until the outputs are equivalent.

3. Calibrate the loudness of the auditory cue. Calibrating the tone intensity requires a decibel meter and the Med-Test software (MED Associates or equivalent from another manufacturer). Med-Test software presents pure tones or white noises of a set frequency and intensity through a given chamber's speaker. Using the $\mathrm{dB}$ meter, measure the speaker output in each chamber (from the same location in every chamber). The reading of the $\mathrm{dB}$ meter should match the selected intensity output. If necessary, the hardware for each chamber can be independently adjusted so that the actual output matches the selected output.

4. Calibrate the load-cell platforms. When using the load cells described in the protocol for automated scoring, it is essential that each load cell output the same voltage for the same amount of transduced movement in each of the conditioning chambers. To calibrate the load cells an oscillator that attaches firmly to the grid floor (MED Associates or equivalent) will be required along with Threshold-Activity software. The oscillator will oscillate at a set frequency causing the Threshold-Activity software to record a waveform. This waveform should be the same for all conditioning chambers and can be adjusted if necessary on the load-cell hardware. Additionally, it is imperative that the load cells be set so that the sensitivity is ideal for recording freezing behavior. In other words, the freezing recorded by the computer cannot be different from that recorded by a trained observer hand scoring the data (for more information on hand scoring data, see UNIT 8.5C). Additionally, when using automated scoring, video recording the rats throughout the experiment is recommended as a backup. If for some reason the automated scoring fails, the videotapes can always be used to hand score all the behavior at a later time.

5. Clean all chambers thoroughly with $1 \%$ acetic acid solution.

6. Program computers for stimulus delivery and data acquisition.

7. Transport the animals in white plastic boxes from their home cages (in the vivarium) into the conditioning room.

8. Place each subject into an individual conditioning chamber and begin videotaping before activating the computer programs.

The number of animals that can be run in a session will depend on the number of conditioning chambers available.

9. Activate the computer programs.

10. At a time point $3 \mathrm{~min}$ after placement in the chambers, present five conditioning trials (70-sec intertrial interval) in which an auditory CS $(2 \mathrm{kHz}, 80 \mathrm{~dB}, 10 \mathrm{sec})$ co-terminates with a footshock US $(1.0 \mathrm{~mA}, 1 \mathrm{sec})$. Measure the freezing behavior throughout the experimental session.

Freezing is quantified by computing the number of observations for each rat that has a value less than the freezing threshold (load-cell activity $=10$ ). An observation is scored as freezing if it falls within a continuous group of at least five observations that are all less than the freezing threshold. Thus, freezing is only scored if the rat is immobile for 
at least 1 sec. Freezing data is converted to a percentage of total observations, which is a probability estimate that is amenable to analysis with parametric statistics. It is suggested that these values be analyzed using analysis of variance (ANOVA) and posthoc comparisons using Fishers LSD tests be performed if a significant overall $F$ ratio is obtained. All data are typically represented as means \pm SEMs.

11. Remove the animals $60 \mathrm{sec}$ after the final shock.

12. Transport the animals back to their home cages.

13. Clean each chamber with $1 \%$ acetic acid solution thoroughly and reset all computer programs.

14. Obtain the next set of animals to be conditioned, and repeat steps 7 to 13 .

15. Turn off equipment at the end of the day.

Day 2: Extinction to an auditory $C S$

Twenty-four hours after conditioning, animals are extinguished to the auditory CS in context B.

16. Turn on all equipment and calibrate again (see steps 2 to 4 for calibration).

17. Clean all chambers thoroughly with $1 \%$ ammonium hydroxide solution.

18. Program computers for stimulus delivery and data acquisition.

19. Transport the animals in black plastic boxes from their home cages into the conditioning room.

20. Place each subject into an individual conditioning chamber.

21. Activate the computer programs.

22. At a time point 3 min after placement in the chambers present 30 auditory CSs $(10 \mathrm{sec} ; 80 \mathrm{~dB}, 70 \mathrm{sec}$ intertrial interval); no USs are delivered during this session. Measure the freezing behavior throughout the experimental session (see step 10).

23. Remove the animals $60 \mathrm{sec}$ after the final CS.

24. Transport the animals back to their home cages.

25. Clean each chamber with $1 \%$ ammonium hydroxide solution thoroughly and reset all computer programs.

26. Obtain the next set of animals to be extinguished and repeat steps 19 to 25 .

27. Turn off equipment at the end of the day.

IMPORTANT NOTE: For NO-EXT controls, the animals are exposed to context B for equivalent amount of time without tone presentations. For experiments with multiple groups per extinction condition (EXT and NO-EXT), the order of the groups should be counterbalanced (for further explanation see the Commentary section).

Day 3: Retention test to an auditory $C S$

Twenty-four hours after extinction, all of the animals are tested to the auditory CS in context B.

28. Turn on all equipment and calibrate again (see steps 2 to 4 for calibration).

29. Clean all chambers thoroughly with $1 \%$ ammonium hydroxide solution.

30. Program computers for stimulus delivery and data acquisition.

\subsection{6}


31. Transport the animals in black plastic boxes from their home cages into the conditioning room.

32. Place each subject into an individual conditioning chamber.

33. Activate the computer programs.

34. At a time point 2 min after placement in the chambers, present 30 auditory CSs (10 sec; $80 \mathrm{~dB}, 70 \mathrm{sec}$ intertrial interval); no USs are delivered during this session. Measure the freezing behavior throughout the experimental session (see step 10).

35. Transport the animals back to their home cages.

36. Clean each chamber with $1 \%$ ammonium hydroxide solution thoroughly and reset all computer programs.

37. Obtain the next set of animals to be tested, and repeat steps 31 to 36 .

38. Turn off all equipment at the end of the day.

\section{CONTEXT-SPECIFIC FEAR EXTINCTION AND RENEWAL}

In the renewal design described here, a third context (context $\mathrm{C}$ ) is used in which all chamber houselights and ventilation fans are turned off. The chambers are cleaned with $70 \%$ ethanol. In addition, stainless steel pans containing a thin layer of the same solution are placed underneath the grid floors before the rats are placed inside to provide a distinct odor. Black plastic floor mats are placed above grid floors. Rats are transported to this context in white plastic boxes with bedding.

After extinction, rats exhibit little fear to a CS in the extinction context, but show a return of fear to that CS in a context in which the CS has not been extinguished. This renewal of fear indicates that extinction is context-specific. This context specificity is modulated by the hippocampus. Inactivation of the hippocampus during either extinction or testing results in generalization to the context (Bouton et al., 2006). There are several different ways the conditioning, extinction, and test contexts can be arranged to observe renewal (i.e., ABA, ABC, AAB). The design of each type of renewal procedure is shown in Table 8.23.2. It takes 3 days to perform these procedures. Rats that do not undergo extinction procedures can serve as a control to compare fear responses to groups that received extinction procedures. For example, when freezing is tested in the extinction context, groups that have been extinguished should display low levels of freezing, whereas nonextinguished controls will display high levels of freezing. When an extinction group is tested in a novel context different from the one they were extinguished in, they will display elevated levels of freezing indicative of fear renewal. The degree of renewal can be compared to rats that did not undergo extinction. For materials, see the Basic Protocol.

\section{Day 1: Conditioning to an auditory CS in a novel context}

1. Follow steps 1 to 15 of day 1 in the Basic Protocol.

\section{Day 2: Extinction to an auditory $C S$}

Twenty-four hours after conditioning, animals are extinguished to the auditory CS in context B.

2. Turn on all equipment and calibrate again (see steps 2 to 4 of the Basic Protocol for calibration).

3. Clean all chambers thoroughly with solution according to Table 8.23.2 depending on context.

4. Follow steps 19 to 27 of day 2 in the Basic Protocol.

Behavioral

Neuroscience

8.23.7

Supplement 47 


\begin{tabular}{|c|c|c|c|}
\hline \multicolumn{4}{|l|}{ ABA vs. AAA } \\
\hline Design & Conditioning & Extinction & Test \\
\hline ABA (DIFF) & $\mathrm{AT}+$ & $\mathrm{BT}-, \mathrm{A}-$ & AT- \\
\hline AAA (SAME) & $\mathrm{AT}+$ & $\mathrm{AT}-, \mathrm{B}-$ & AT- \\
\hline \multicolumn{4}{|l|}{ AAB vs. $\mathrm{ABB}$} \\
\hline Design & Conditioning & Extinction & Test \\
\hline AAB (DIFF) & $\mathrm{AT}+$ & $\mathrm{AT}-, \mathrm{B}-$ & $\mathrm{BT}-$ \\
\hline ABB (SAME) & $\mathrm{AT}+$ & $\mathrm{BT}-, \mathrm{A}-$ & $\mathrm{BT}-$ \\
\hline \multicolumn{4}{|l|}{ ABC vs. ACC } \\
\hline Design & Conditioning & Extinction & Test \\
\hline ABC (DIFF) & $\mathrm{AT}+$ & $\mathrm{BT}-, \mathrm{C}-$ & $\mathrm{CT}-$ \\
\hline ACC (SAME) & $\mathrm{AT}+$ & $\mathrm{CT}-, \mathrm{B}-$ & $\mathrm{CT}-$ \\
\hline
\end{tabular}

ALTERNATE PROTOCOL 2

\section{ALTERNATE PROTOCOL 3}

Fear Extinction in Rodents

8.23.8

\section{Day 3: Retention test to an auditory CS}

Twenty-four hours after extinction, all of the animals are tested to the auditory CS in context B.

5. Turn on all equipment and calibrate again (see steps 2 to 4 of the Basic Protocol for calibration).

6. Clean all chamber thoroughly with solution according to Table 8.23.2 depending on context.

7. Follow steps 30 to 38 of the Basic Protocol.

\section{SPONTANEOUS RECOVERY OF FEAR MEMORY IN RATS}

Extinguished fear memory recovers with the passage of time. Testing for recovery of extinguished fear memory can be easily conducted with any extinction design. Simply repeat retention test procedures in the extinction context 7, 14, or 21 days after the first test session. The duration after extinction before retention testing is variable and is determined by the experimenter based on what retention interval is of most interest.

To test for spontaneous recovery of fear memory, perform steps 1 to 38 of the Basic Protocol at various time intervals. Selection of the most appropriate time to perform the retention test will depend on the interval between extinction training and retention testing as decided by the experimenter.

\section{REINSTATEMENT OF FEAR MEMORY AFTER EXTINCTION}

Exposure to a single presentation of the US after extinction can cause a recovery of conditioned fear to the CS during a later retention test. This is a 4-day procedure with the reinstatement shock session on day 3 and the retention test on day 4 . The general design of the experiment is summarized in Table 8.23.3. A group of rats in which fear is extinguished, but which does not receive reinstatement shock can be used to control for nonassociative sensitization of shock. For materials, see the Basic Protocol. 
Table 8.23.3 Reinstatement of Fear Memory Experimental Design (Alternate Protocol 3) ${ }^{a}$

\begin{tabular}{lcccc}
\hline Design & Conditioning & Extinction & Reinstatement & Test \\
\hline DIFF & AT + & AT,- B - & B + & AT - \\
SAME & AT + & AT,- B - & A + & AT - \\
NO-SHOCK & AT + & AT,- B - & A- or B- & AT - \\
\hline
\end{tabular}

${ }^{a}$ SAME and DIFF denote whether reinstatement and test occur in the same or different contexts. Other notations are the same as described in Tables 8.23.1 and 8.23.2.

\section{Day 1: Conditioning to an auditory CS in a novel context}

1. Perform steps 1 to 15 of the Basic Protocol.

\section{Day 2: Extinction to an auditory $C S$}

Twenty-four hours after conditioning, animals are extinguished to the auditory CS in context B.

2. Perform steps 16 to 27 of the Basic Protocol.

Day 3: Exposure to single presentation of US

Twenty-four hours after extinction, all of the animals are exposed to a single presentation of the US.

3. Clean all chambers thoroughly according to Table 8.23.3 depending on context.

4. Program computers for stimulus delivery and data acquisition.

5. Transport the animals from their home cages in the vivarium into the conditioning room.

6. Place each subject into an individual conditioning chamber and begin videotaping before activating the computer programs.

7. Activate the computer programs.

8. At a time point $3 \mathrm{~min}$ after placement in the chambers, present one footshock US $(1.0 \mathrm{~mA}, 1 \mathrm{sec})$. Freezing behavior is measured throughout the experimental session (see step 10 of the Basic Protocol).

9. Remove the animals $60 \mathrm{sec}$ after the shock.

10. Transport the animals back to their home cages.

11. Clean each chamber thoroughly and reset all computer programs.

12. Obtain the next set of animals to be conditioned and repeat steps 5 to 11 .

13. Turn off equipment at the end of the day.

Day 4: Retention test to an auditory $C S$

14. Repeat steps 28 to 38 of the Basic Protocol.

\section{COMMENTARY}

\section{Background Information}

In the laboratory, memories of traumatic experiences can be modeled using Pavlovian fear conditioning in rats. For this procedure, an innocuous conditioned stimulus (CS), such as a tone, is presented with an aversive unconditioned stimulus (US), such as a mild electric shock. After conditioning, the CS elicits a learned fear response, which includes increases in heart rate, blood pressure, and stress
Behavioral Neuroscience

\subsection{9}

Supplement 47 
hormone release (Fanselow and Poulos, 2005). A unique feature of fear memory is that it can be acquired with as little as one exposure, and can persist for a lifetime (Maren, 2005). However, if the CS is repeatedly presented in the absence of the US, conditioned fear responses begin to diminish, a phenomenon known as extinction. Rather than an erasure of the previous CS-US memory, a new association is formed between the CS and absence of US during extinction that effectively suppresses the previously acquired CR (Ji and Maren, 2007). Evidence of fear renewal in which fear memories can be unmasked in a context different from that of the extinction context supports the extinction association (Bouton et al., 2006). In addition, the extinguished CRs can be recalled over time (Quirk, 2002) or if the subject is exposed to the US without the CS (Ji and Maren, 2007).

It is widely accepted that the amygdala is the site of the synaptic plasticity that mediates both the acquisition and the inhibition of new fears after conditioning and extinction, respectively (Bruchey et al., 2007; Corcoran and Quirk, 2007). The amygdala consists of several nuclei, including the central nucleus (CEA) and the basolateral complex (BLA), which itself is composed of the lateral (LA), basomedial (BM), and basolateral (BL) nuclei (Maren, 2001). According to the current models of fear circuitry, the BLA is the site at which sensory information about the CS and US converge during initial fear learning, and the acquisition of fear responses occurs. Receiving input both directly and indirectly from the BLA, the CEA primarily mediates the expression of the fear response; however, the CEA is also necessary for acquisition and long-term storage of fear memories (LeDoux et al., 1988; Wilensky et al., 2006; Zimmerman et al., 2007).

As with fear conditioning, the BLA is also involved in forming extinction memories (Bruchey et al., 2007). However, unlike fear conditioning, extinction may involve local inhibitory neurons in the BLA and the CEA, as well as GABAergic inhibitory interneurons located between the two nuclei through which suppression of BLA excitatory projection neurons occurs (Royer and Pare, 2002). As such, extinction can be viewed as parallel learning that does not affect previous plasticity but rather reflects an increase in plasticity in the GABAergic inhibitory interneurons, due to glutamatergic input from the BLA. Additionally, the amygdala receives information re- garding the auditory CS during extinction (Teich et al., 1989), although the route by which this information reaches the amygdala is not as clear as it is for fear conditioning. Beyond the amygdala, other neural structures may be involved in extinction such as the ventromedial prefrontal cortex (vmPFC) and the hippocampus, both of which have interconnections with the amygdala (Quirk and Mueller, 2008). These structures appear to modulate the inhibition of CRs and the specifics of the surrounding environment, respectively (Bruchey et al., 2007; Ji and Maren, 2007).

Considerable evidence has shown that extinction memories are labile; they dissipate with time and change in context. In fact, although extinction effectively retards conditional responding, it is a relatively transitory phenomenon that is context-specific. Various studies have demonstrated that if an animal is trained in context $\mathrm{A}$, extinguished in context $\mathrm{B}$, and tested in context $\mathrm{C}$, the animal will show a reliable increase in freezing during testing relative to extinction training (Bouton et al., 2006). This renewal effect clearly illustrates that the environment is important in determining whether the extinction memory is retrieved. The role of context in the regulation of CR suppression is also evident in another context effect: spontaneous recovery, where the fear response will gradually appear after the passage of time when tested in the same context in which extinction occurred (Quirk, 2002). Moreover, a final example of the context specificity of extinction is reinstatement, or the appearance of the CR in response to the US alone in a related context. All of these effects suggest that excitatory and inhibitory memories are established during conditioning and extinction, respectively, and that context serves as a retrieval cue to determine which memory is expressed when the CS is presented.

Not surprisingly, the hippocampus, a structure that encodes contextual and temporal information, may be critical in mediating the context's control on extinction and therefore have a role in the consolidation of extinction memories (Ji and Maren, 2007; Quirk and Mueller, 2008). Inactivation of the hippocampus prevents the typical renewal effect to the CS when it is presented in a context different from that in which extinction took place (Corcoran and Maren, 2001, 2004; Corcoran et al., 2005). As mentioned previously, the vmPFC may be involved in the contextual modulation of extinction insofar as it receives 
projections from the hippocampus (Bruchey et al., 2007). In turn, the vmPFC regulates neuronal activity in the amygdala through two distinct pathways that act in an opposing manner: the infralimbic (IL) pathway, which inhibits CR expression, and the prelimbic (PL) pathway, which drives the expression of CRs (Vidal-Gonzalez et al., 2006; Corcoran and Quirk, 2007; Quirk and Mueller, 2008). These two pathways may provide a way in which the hippocampus can dictate whether the fear responses are either inhibited or expressed, depending on the context.

It is apparent that extinction forms a separate memory from conditioning, yet both learning processes undergo similar plastic changes resulting in stable memories. Understanding the neural structures and processes involved in extinction is critical for developing effective treatments for inhibiting pathological fear in humans.

\section{Critical Parameters and Troubleshooting}

\section{The context specificity of extinction}

As mentioned previously, extinction is highly context-dependent, as demonstrated by renewal experiments (for a review see Bouton, 1993). It is therefore essential to ensure that all of the contexts are very distinct from one another. If the distinction between contexts is inadequate, the rats will often display generalized fear when placed into a context that should not be innately fearful. For example, when completing a renewal experiment with the ABA design, following conditioning (designated as $\mathrm{AT}+$, meaning context $\mathrm{A}$ with a tone $\mathrm{T}$, and the presence of a footshock + ), when rats are placed into the $\mathrm{B}$ context for extinction (designated as BT-, meaning context $\mathrm{B}$ with a tone $\mathrm{T}$, but with no footshock -), they may freeze before any tones are presented, even though they have no previous experience with this context. The likely cause of this is context generalization, which can be caused by insufficient cleaning of the chamber between squads of rats or similarities between the contextual properties. This is easily eliminated by ensuring that the contexts are sufficiently different and by cleaning the chambers thoroughly between tests. However, even with these precautions some contextual generalization may occur, especially when higher shock intensities are used during conditioning.

\section{Timing}

The delay between conditioning and extinction can have dramatic consequences on the effectiveness of extinction. Maren and Chang (2006) have shown that extinction testing only 15 min after conditioning drastically reduces the retention of extinction. In fact, the effect is so dramatic that the animals receiving extinction 15 min after conditioning show levels of fear that are not significantly different from the unextinguished (NO-EXT) controls. However, waiting longer after conditioning before beginning extinction, such as 24 hours, can increase the retention of extinction. It is therefore essential to consider the timing delay between conditioning and extinction when designing experiments that are aimed at increasing the retention of extinction.

\section{Counterbalancing}

As noted in the renewal protocol (Alternate Protocol 1), it is important to counterbalance the contexts (A or B), the order of groups in each phase (SAME or DIFF), and the chambers in which the rats are trained, tested, and extinguished. Counterbalancing the contexts ensures that the measured level of fear is not being differentially influenced by the contexts. For the ABA (DIFF) renewal protocol described above, counterbalancing entails running half of the animals in the ABA design and half of them in a BAB design. The results can then be collapsed across those groups.

The time of day can influence context (Bouton, 1993). Counterbalancing the order of groups eliminates the variable of time. To do this, simply alternate the groups (DIFF and SAME).

If multiple chambers are being used for the experiment, it is essential to ensure that the chambers that each experimental group is in are counterbalanced. In other words, make sure each chamber is used by each group of animals so that all of the SAME animals are not in one chamber, and all of the DIFF animals are not in another chamber. This will control for any variable differences between the chambers.

\section{Within-subjects experimental design}

To this point, only between-subjects experimental designs have been discussed. Under certain circumstances, a within-subjects experimental design may be more appropriate. In such an extinction protocol, the rats would be trained to multiple discrete CSs such as a pure tone and white noise burst. The discrete CSs would then be extinguished in different contexts and a retrieval test would be performed for each CS in both of the extinction contexts. For example, train the rats to both cues in
Behavioral Neuroscience

\subsubsection{1}

Supplement 47 
context $\mathrm{A}$, extinguish the pure tone $\mathrm{CS}$ in context $\mathrm{B}$ and the white noise $\mathrm{CS}$ in context $\mathrm{C}$, and finally test for retrieval to each CS in both contexts B and C. Such a protocol allows for the direct comparison of the $\mathrm{CR}$ between tests within the same subjects. For a more detailed example of a within-subjects protocol, see Hobin et al. (2003).

\section{Calibration and scoring}

Inter-chamber reliability is essential to minimizing error within experimental groups. It is therefore necessary to calibrate the conditioning chambers before each experiment. Variations in tone intensity (CS), footshock intensity (US), or load cell output [activity or freezing (CR)] between chambers add additional variables that make interpreting the data difficult (see steps 3 to 5 of the Basic Protocol for details).

\section{Pavlovian fear conditioning}

For a review of the critical parameters for Pavlovian fear conditioning, see UNIT 8.5C.

Depending on the aspect of extinction learning and memory being studied, experimental manipulations can be performed before, during, or after a specific phase of training and/or testing to isolate the neural substrates involved in that process. For instance, if examining the effects of a drug during extinction learning but not conditioning, it would be appropriate to apply the drug prior to the extinction session, whereas if the effects of a drug on extinction memory recall were of interest, the drug would only be applied prior to the recall test.

When considering the number of CS-US pairings and US intensity to use during conditioning, pilot studies should be undertaken to optimize conditions in each laboratory. For example, the authors' laboratory typically uses five CS-US pairings and a US intensity of $1.0 \mathrm{~mA}$ to reliably produce robust fear.

Most methodologies described here for rats generalize to mice, although there are species and strain differences (e.g., in fear-related behaviors, as well as in pain perception, hearing, etc.). All those should be taken into consideration when designing experiments (Bolivar et al., 2001).

\section{Anticipated Results}

\section{Basic Protocol}

During conditioning, both groups (EXT and NO-EXT) should show very low levels of freezing before the first tone-shock presentation. Following tone-shock presentations the levels of freezing should increase significantly in both groups (see Fig. 8.23.3A). During extinction, the EXT group should show low levels of freezing before the first tone presentation. Following the first tone presentation the EXT group should show a drastically enhanced freezing response. Following each subsequent tone presentation, the levels of freezing should slowly decrease until they reach near baseline levels by the end of the extinction session. The NO-EXT group should show low levels of freezing throughout the extinction session. The level of freezing recorded by the load cells may start to increase slightly for the NO-EXT group towards the end of the extinction session as animals become less active (this does not look like freezing when observing the behavior of the rats; see Fig. 8.23.3B). During the test phase of the experiment, both EXT and NO-EXT groups should show low levels of fear before the tone onset. Following the tone onset, the EXT group may show a slight increase in freezing but it will rapidly return to baseline. The NO-EXT group, however, should show very robust levels of freezing following the tone onset and remain significantly higher than the EXT group through the majority of the test (see Fig. 8.23.3C).

\section{Alternate Protocol 1: Renewal of fear}

Results during the conditioning session should be identical to those described for the Basic Protocol and in Figure 8.23.3. Results for the ABA (DIFF) group during the extinction phase should also be the same as those described above. Freezing levels for the AAA (SAME) group should be the same as those described above for the ABA (DIFF) group, except that the rats will show high levels of freezing even before the first tone presentation arising from their fear to the conditioning context (see Fig. 8.23.4A). During the exposure to the other context rats in the ABA (DIFF) group will show high levels of fear when returned to A(context A with no footshock), but will show extinction throughout the session eventually returning to near baseline levels of freezing. Rats in the AAA (SAME) group will show low levels of freezing when placed in the B- context (context B with no footshock), similar to that described for the NO-EXT group during extinction in the Basic Protocol (see Fig. 8.23.4B). During the test phase, both groups should show low levels of freezing when initially placed in the chamber. Following the tone onset, rats in the ABA (DIFF) groups should show significantly more 

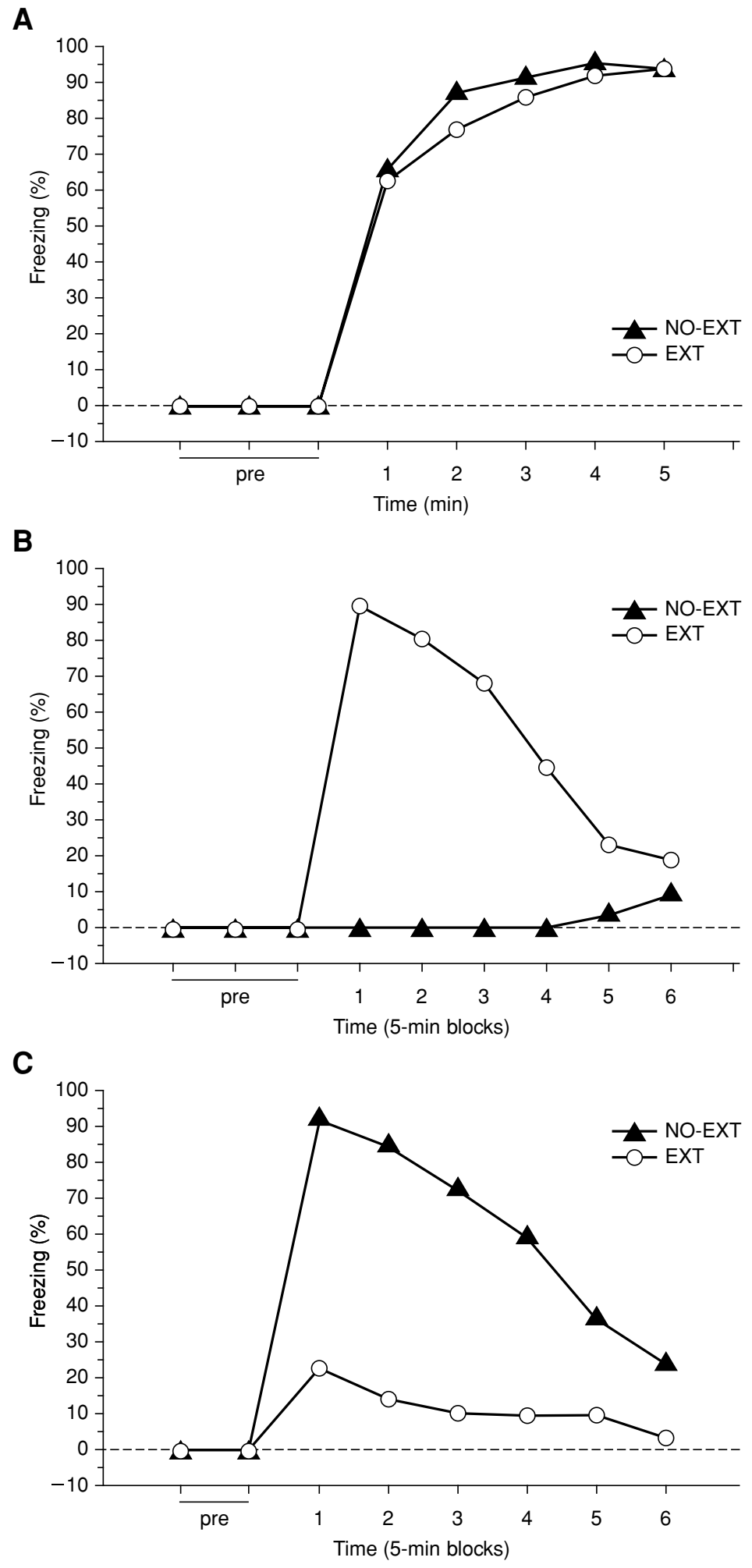

Figure 8.23.3 Idealized graphs showing anticipated results of the Basic Protocol. Average percentage of freezing during (A) training (conditioning), (B) extinction, and (C) retention testing. Abbreviations: EXT, extinction group; NO-EXT, no-extinction control group; pre, baseline freezing before auditory CS.

Behavioral

Neuroscience

8.23.13 


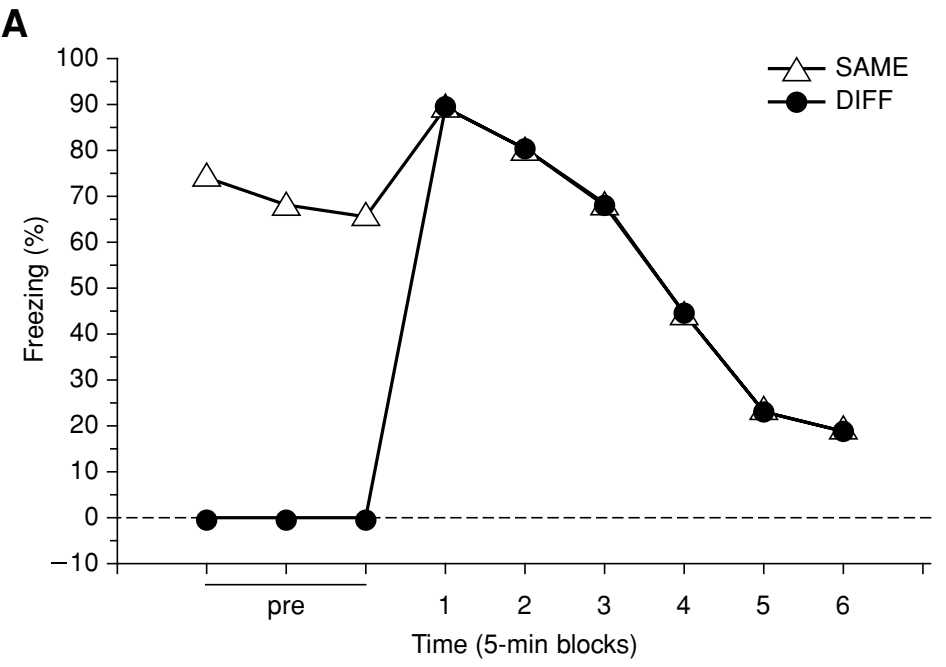

B

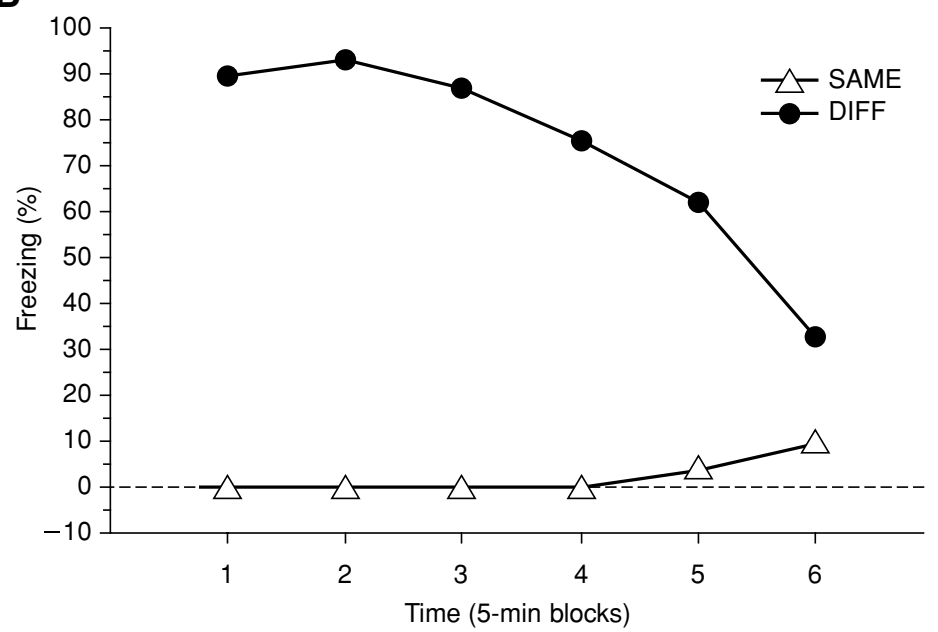

C

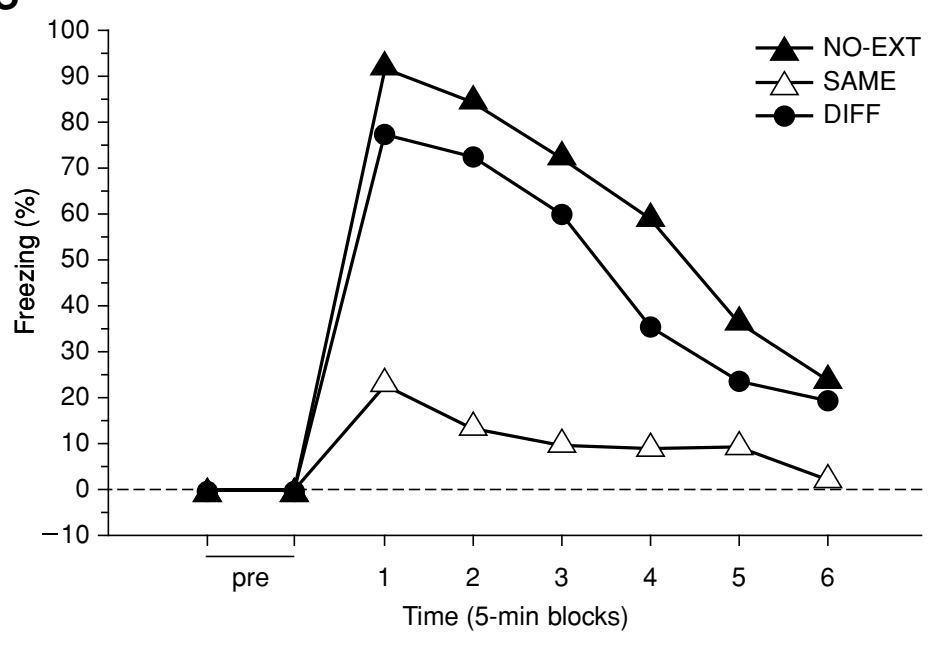

Figure 8.23.4 Idealized graphs showing anticipated results of Alternate Protocol 1. Average percentage of freezing during (A) extinction, (B) context exposure, and (C) retention testing (renewal). Legends: DIFF, ABA group; SAME, AAA group. A non-extinguished control group (NO-EXT) is shown for comparison during the retention test. 
Retention Test: Spontaneous Recovery

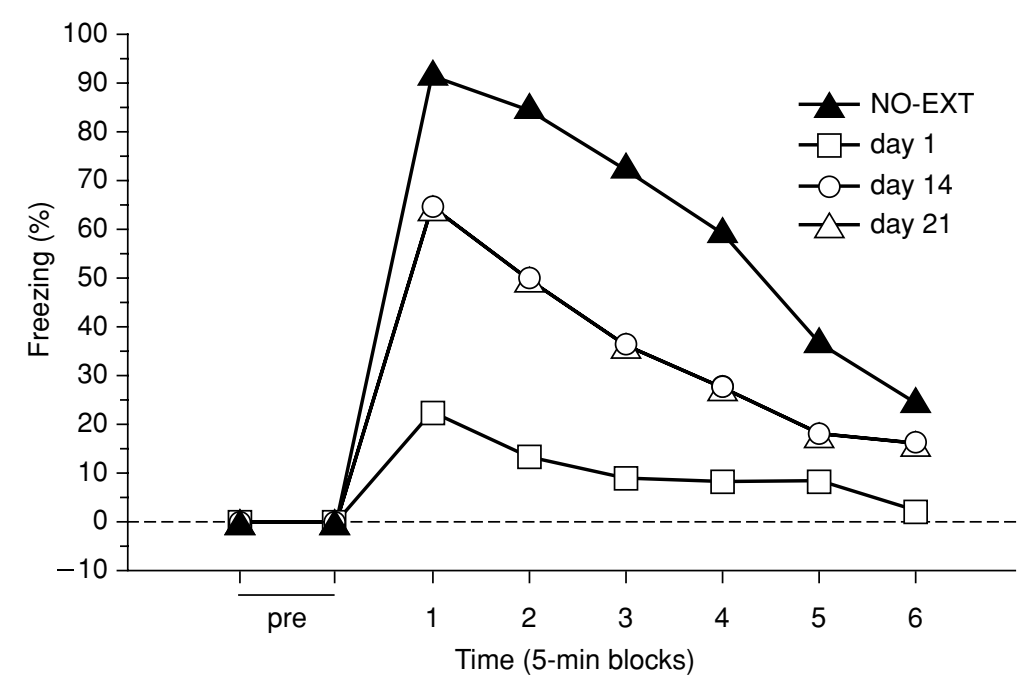

Figure 8.23.5 Idealized graph showing anticipated results of Alternate Protocol 2. Average percentage of freezing during the retention test performed after 1, 14, or 21 days. A non-extinguished control group (NO-EXT) is shown for comparison during the retention test.

Retention Test: Reinstatement

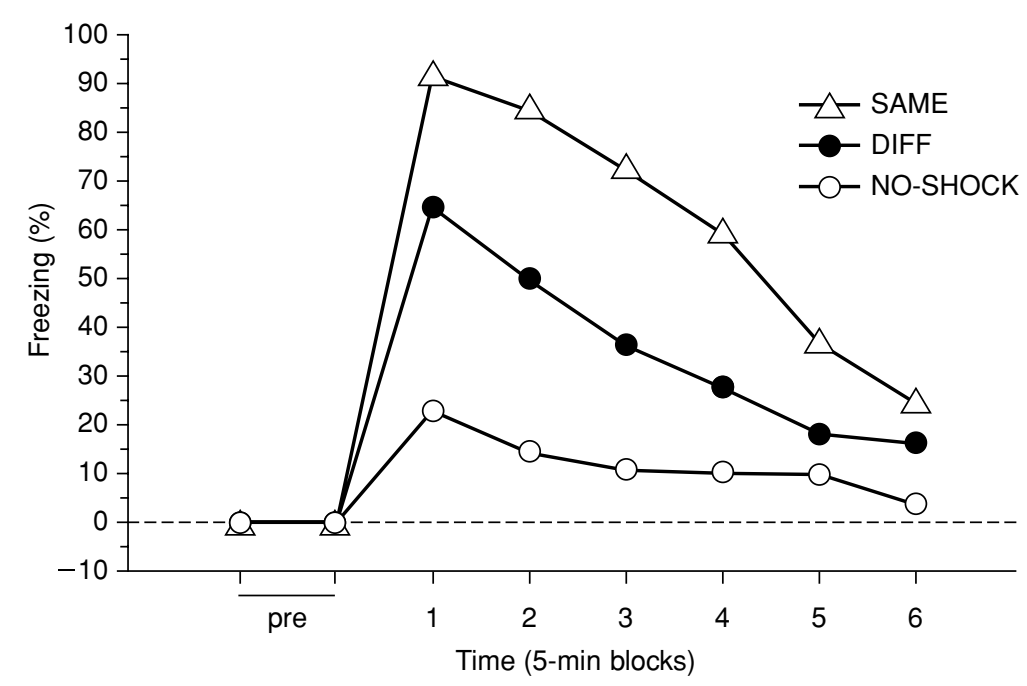

Figure 8.23.6 Idealized graph showing anticipated results of Alternate Protocol 3. Percentage of freezing during the tone retention test in rats tested in the same (SAME) or different (DIFF) context as the one in which reinstatement shock was delivered. An extinguished control group that did not receive reinstatement shocks (NO-SHOCK) is shown for comparison.

Behavioral

Neuroscience

8.23.15 
freezing than rats in the AAA (SAME) group (see Fig. 8.23.4C). Rats in the AAA (SAME) group should look similar to the EXT group during test in the Basic Protocol.

\section{Alternate Protocol 2: Spontaneous recovery of fear}

Results during the conditioning, extinction, and first retention test should look identical to those described above for the Basic Protocol. When tested for retention 14 or 21 days after the initial retention test, rats in the EXT group should show higher levels of freezing than they did during the initial retention test (see Fig. 8.23.5).

\section{Alternate Protocol 3: Reinstatement of fear}

Conditioning and extinction in both groups (DIFF and SAME) should look identical to that described for the AAA (SAME) group in Alternate Protocol 1. During testing, both groups should show low levels of freezing. Following tone onset, rats in the SAME group should show substantially more freezing than rats in the DIFF group (see Fig. 8.23.6). Rats in the DIFF group may exhibit more freezing than NO-SHOCK rats due to nonassociative sensitization of fear.

\section{Time Considerations}

The number of days required for each protocol is listed within the protocol. When designing an experiment and the number of animals to run each day, keep in mind that the extinction sessions will take the longest and the amount of time between sessions for each animal should be kept constant. Typically, 32 to 48 rats per experiment with eight rats per experimental group are used to obtain to required statistical power. In the authors' laboratory, eight rats per squad are run simultaneously, and all squads are run each day of the experiment. However, the number of rats per squad and number of squads will depend on the number of chambers available to the experimenter.

\section{Acknowledgements}

This unit was supported by grants from the NIH (R01MH065961; R01MH073655) to S.M.

\section{Literature Cited}

Bolivar, V.J., Pooler, O., and Flaherty, L. 2001. Inbred strain variation in contextual and cued fear conditioning behavior. Mamm. Genome 12:651656.

Bouton, M.E. 1993. Context, time, and memory retrieval in the interference paradigms of Pavlovian learning. Psychol. Bull. 114:80-99.
Bouton, M.E., Westbrook, R.F., Corcoran, K.A., and Maren, S. 2006. Contextual and temporal modulation of extinction: behavioral and biological mechanisms. Biol. Psychiat. 60:352-360.

Bruchey, A.K., Shumake, J., and Gonzalez-Lima, F. 2007. Network model of fear extinction and renewal functional pathways. Neuroscience 145:423-437.

Corcoran, K.A., Desmond, T.J., Frey, K.A., and Maren, S. 2005. Hippocampal inactivation disrupts the acquisition and contextual encoding of fear extinction. J. Neurosci. 25:8978-8987.

Corcoran, K.A. and Maren, S. 2001. Hippocampal inactivation disrupts contextual retrieval of fear memory after extinction. J. Neurosci. 21:17201726.

Corcoran, K.A. and Maren, S. 2004. Factors regulating the effects of hippocampal inactivation on renewal of conditional fear after extinction. Learn. Mem. 11:598-603.

Corcoran, K.A. and Quirk, G.J. 2007. Activity in prelimbic cortex is necessary for the expression of learned, but not innate, fears. J. Neurosci. 27:840-844.

Davis, M. 2001. Fear-potentiated startle in rats. Curr. Protoc. Neurosci. 14:8.11A.1-8.11A.11.

Fanselow, M.S. and Poulos, A.M. 2005. The neuroscience of mammalian associative learning. Annu. Rev. Psychol. 56:207-234.

Hermans, D., Craske, M.G., Mineka, S., and Lovibond, P.F. 2006. Extinction in human fear conditioning. Biol. Psychiat. 60:361-368.

Hobin, J.A., Goosens, K.A., and Maren, S. 2003. Context-dependent neuronal activity in the lateral amygdala represents fear memories after extinction. J. Neurosci. 23:8410-8416.

Ji, J. and Maren, S. 2007. Hippocampal involvement in contextual modulation of fear extinction. Hippocampus 17:749-758.

LeDoux, J.E., Iwata, J., Cicchetti, P., and Reis, D.J. 1988. Different projections of the central amygdaloid nucleus mediate autonomic and behavioral correlates of conditioned fear. J. Neurosci. 8:2517-2529.

Maren, S. 2001. Neurobiology of Pavlovian fear conditioning. Annu. Rev. Neurosci. 24:897-931.

Maren, S. 2005. Synaptic mechanisms of associative memory in the amygdala. Neuron 47:783786.

Maren, S. and Chang, C.H. 2006. Recent fear is resistant to extinction. Proc. Natl. Acad. Sci. U.S.A. 103:18020-18025.

Quirk, G.J. 2002. Memory for extinction of conditioned fear is long-lasting and persists following spontaneous recovery. Learn. Mem. 9:402-407.

Quirk, G.J. and Mueller, D. 2008. Neural mechanisms of extinction learning and retrieval. Neuropsychopharmacology 33:56-72.

Royer, S. and Pare, D. 2002. Bidirectional synaptic plasticity in intercalated amygdala neurons and the extinction of conditioned fear responses. Neuroscience 115:455-462. 
Teich, A.H., McCabe, P.M., Gentile, C.C., Schneiderman, L.S., Winters, R.W., Liskowsky, D.R., and Schneiderman, N. 1989. Auditory cortex lesions prevent the extinction of Pavlovian differential heart rate conditioning to tonal stimuli in rabbits. Brain. Res. 480:210-218.

Vidal-Gonzalez, I., Vidal-Gonzalez, B., Rauch, S.L., and Quirk, G.J. 2006. Microstimulation reveals opposing influences of prelimbic and infralimbic cortex on the expression of conditioned fear. Learn. Mem. 13:728-733.

Wilensky, A.E., Schafe, G.E., Kristensen, M.P., and LeDoux, J.E. 2006. Rethinking the fear circuit: the central nucleus of the amygdala is required for the acquisition, consolidation, and expression of Pavlovian fear conditioning. J. Neurosci. 26:12387-12396.

Woods, A.M. and Bouton, M.E. 2006. D-cycloserine facilitates extinction but does not eliminate renewal of the conditioned emotional response. Behav. Neurosci. 120:1159-1162.

Zimmerman, J.M., Rabinak, C.A., McLachlan, I.G., and Maren, S. 2007. The central nucleus of the amygdala is essential for acquiring and expressing conditional fear after overtraining. Learn. Mem. 14:634-644.
Behavioral Neuroscience

8.23.17 